Adv. Studies Theor. Phys., Vol. 7, 2013, no. 3, 127 - 130 HIKARI Ltd, www.m-hikari.com

\title{
On Hamilton Paths in Grid Graphs
}

\author{
Anna Gorbenko \\ Department of Intelligent Systems and Robotics \\ Ural Federal University \\ 620083 Ekaterinburg, Russia \\ gorbenko.ann@gmail.com \\ Vladimir Popov \\ Department of Intelligent Systems and Robotics \\ Ural Federal University \\ 620083 Ekaterinburg, Russia \\ Vladimir.Popov@usu.ru
}

\begin{abstract}
In this paper we consider an approach to solve the Hamilton path problem for grid graphs. This approach is based on an explicit reduction from the problem to the satisfiability problem.
\end{abstract}

PACS: 07.05.Fb, 07.05.Mh, 07.05.Tp

Keywords: Hamilton path, grid graph, NP-complete, satisfiability, vacuum cleaning robot

Gridworlds are extensively used for design of experiments as models of real world. In particular, grid graphs are popular testbeds for different hard problems. In particular, grid graphs used for investigation of robotic planning problems (see e.g. [1]). For instance, grid graph can be considered as a map of some indoor environment. Vacuum cleaning robot needs cover the environment. The robot must visit each vertex only once to prevent a damage of the floor. It is clear that we can consider the Hamilton path problem (HP) for grid graphs as a model for this robotic problem. In this paper we consider an approach to solve HP. Note that HP is NP-complete [2]. Encoding different hard problems as instances of SAT has caused considerable interest (see e.g. $[3]-[15])$. We consider an explicit reduction from HP to the satisfiability problem. 
Let $G=(V, E)$ be a grid graph. Clearly, we can consider $G$ as a part of two-dimensional integer grid. We assume that $V=\left\{G\left(i_{1}, j_{1}\right), \ldots, G\left(i_{n}, j_{n}\right)\right\}$, $(G(i, j), G(s, t)) \in E \Leftrightarrow|i-s|+|j-t|=1 \wedge\{G(i, j), G(s, t)\} \subseteq V$. Also, we can assume that $1 \leq i_{k} \leq p, 1 \leq j_{k} \leq q$, for any $1 \leq k \leq n$. Let

$$
\begin{aligned}
& \varphi[1]=\wedge_{1 \leq k \leq n} \vee_{1 \leq i \leq p} \vee_{1 \leq j \leq q} g[k, i, j], \\
& \varphi[2]=\wedge_{1 \leq k \leq n,} \quad(\neg g[k, i[1], j[1]] \vee \neg g[k, i[2], j[2]]), \\
& 1 \leq i[1] \leq p, \\
& 1 \leq i[2] \leq p, \\
& 1 \leq j[1] \leq q \\
& 1 \leq j[2] \leq q, \\
& |i[1]-i[2]|+|j[1]-j[2]| \neq 0 \\
& \varphi[3]=\wedge_{1 \leq k \leq n,} \neg g[k, i, j], \\
& 1 \leq i \leq p \text {, } \\
& 1 \leq j \leq q, \\
& G(i, j) \notin E \\
& \varphi[4]=\wedge_{1 \leq k \leq n-1, \quad} \quad(\neg g[k, i[1], j[1]] \vee \neg g[k+1, i[2], j[2]]), \\
& 1 \leq i[1] \leq p, \\
& 1 \leq i[2] \leq p, \\
& 1 \leq j[1] \leq q \text {, } \\
& 1 \leq j[2] \leq q, \\
& |i[1]-i[2]|+|j[1]-j[2]| \neq 1 \\
& \xi=\wedge_{i=1}^{4} \varphi[i] .
\end{aligned}
$$

Theorem. There is a Hamilton path in $G$ if and only if $\xi$ is satisfiable.

Proof. Let $\xi=1$. By definition, in this case, $\varphi[i]=1$, for all $1 \leq i \leq 4$. Since $\varphi[1]=1$, it is clear that for any $1 \leq k \leq n$, there are $i$ and $j$ such that $1 \leq$ $i \leq p, 1 \leq j \leq q$, and $g[k, i, j]=1$. In view of $\varphi[2]=1$, it is easy to check that for any $1 \leq k \leq n$, there is only one pair $(i, j) \in\{1,2, \ldots, p\} \times\{1,2, \ldots, q\}$ such that $g[k, i, j]=1$. Therefore, we can assume that for any $1 \leq k \leq n, g[k, i, j]=$ 1 if and only if $(i, j)=(a[k], b[k])$ where $\{(a[1], b[1]),(a[2], b[2]), \ldots,(a[n], b[n])\}$ is some fixed set. Since $\varphi[3]=1$, it is clear that for any $1 \leq k \leq n$, if $g[k, i, j]=1$, then $G(i, j) \in V$. In view of $\varphi[4]=1$, it is easy to see that for all $1 \leq k \leq n-1,1 \leq i[1] \leq p, 1 \leq i[2] \leq p, 1 \leq j[1] \leq q, 1 \leq j[2] \leq q$, if $g[k, i[1], j[1]]=g[k+1, i[2], j[2]]=1$, then $|i[1]-i[2]|+|j[1]-j[2]|=1$. Therefore, if $g[k, i[1], j[1]]=g[k+1, i[2], j[2]]=1$, then $(G(a[k], b[k]), G(a[k+$ $1], b[k+1])) \in E$. So, $G(a[1], b[1]), G(a[2], b[2]), \ldots, G(a[n], b[n])$ is a Hamilton path in $G$. Now, let $G(a[1], b[1]), G(a[2], b[2]), \ldots, G(a[n], b[n])$ be a Hamilton path in $G$. Let $g[k, i, j]=1$ if and only if $i=a[k], j=b[k]$. It is easy to check that $\xi=1$.

It is clear that $\xi$ is a CNF. So, $\xi$ gives us an explicit reduction from HP to SAT. Now, using standard transformations (see e.g. [16]) we can obtain an 


\begin{tabular}{|l|llllll|}
\hline time & N1 & N2 & N3 & R1 & R2 & R3 \\
\hline average & $3.1 \mathrm{~min}$ & $15.2 \mathrm{~min}$ & $1.62 \mathrm{~h}$ & $19 \mathrm{sec}$ & $9.4 \mathrm{~min}$ & $57 \mathrm{~min}$ \\
maximum & $41 \mathrm{~min}$ & $2.7 \mathrm{~h}$ & $11.6 \mathrm{~h}$ & $22 \mathrm{~min}$ & $2.1 \mathrm{~h}$ & $9.36 \mathrm{~h}$ \\
best & $12 \mathrm{sec}$ & $43 \mathrm{sec}$ & $32.3 \mathrm{~min}$ & $7 \mathrm{sec}$ & $28 \mathrm{sec}$ & $19.8 \mathrm{~min}$ \\
\hline
\end{tabular}

Table 1: Experimental results for different reductions to 3SAT where N1 is $\zeta$ for natural instances, N2 is $\gamma[1]$ from [18] for natural instances, N3 is the reduction from [19] for natural instances, $\mathrm{R} 1$ is $\zeta$ for robotic instances, $\mathrm{R} 2$ is $\gamma[1]$ from [18] for robotic instances, R3 is the reduction from [19] for robotic instances.

explicit transformation $\xi$ into $\zeta$ such that $\xi \Leftrightarrow \zeta$ and $\zeta$ is a 3-CNF. Clearly, $\zeta$ gives us an explicit reduction from HP to 3SAT.

We consider our genetic algorithm which was proposed in [17]. We have used heterogeneous cluster for our computational experiments. Each test was runned on a cluster of at least 100 nodes. We have created a generator of natural instances for HP. Also, we have created a generator of instances for $\mathrm{HP}$ which allow us to construct a plan for a vacuum cleaning robot. Selected experimental results are given in Table 1.

ACKNOWLEDGEMENTS. The work was partially supported by Analytical Departmental Program "Developing the scientific potential of high school" 8.1616.2011.

\section{References}

[1] A. Gorbenko, V. Popov, and A. Sheka, Localization on Discrete Grid Graphs, Lecture Notes in Electrical Engineering, 107 (2012), 971-978.

[2] A. Itai, C.H. Papadimitriou, and J.L. Szwarcfiter, Hamilton Paths in Grid Graphs, SIAM Journal on Computing, 11 (1982), 676-686.

[3] A. Gorbenko and V. Popov, On the Longest Common Subsequence Problem, Applied Mathematical Sciences, 6 (2012), 5781-5787.

[4] A. Gorbenko and V. Popov, On the Problem of Sensor Placement, Advanced Studies in Theoretical Physics, 6 (2012) 1117-1120.

[5] A. Gorbenko and V. Popov, The Problem of Selection of a Minimal Set of Visual Landmarks, Applied Mathematical Sciences, 6 (2012), 4729-4732.

[6] A. Gorbenko and V. Popov, The Longest Common Parameterized Subsequence Problem, Applied Mathematical Sciences, 6 (2012), 2851-2855. 
[7] A. Gorbenko and V. Popov, Programming for Modular Reconfigurable Robots, Programming and Computer Software, 38 (2012), 13-23.

[8] A. Gorbenko and V. Popov, The set of parameterized k-covers problem, Theoretical Computer Science, 423 (2012), 19-24.

[9] A. Gorbenko and V. Popov, The Problem of Finding Two Edge-Disjoint Hamiltonian Cycles, Applied Mathematical Sciences, 6 (2012), 6563-6566.

[10] A. Gorbenko and V. Popov, Hamiltonian Alternating Cycles with Fixed Number of Color Appearances, Applied Mathematical Sciences, 6 (2012), 6729-6731.

[11] A. Gorbenko and V. Popov, Footstep Planning for Humanoid Robots, Applied Mathematical Sciences, 6 (2012), 6567-6571.

[12] A. Gorbenko, M. Mornev and V. Popov, Planning a Typical Working Day for Indoor Service Robots, IAENG International Journal of Computer Science, 38 (2011) 176-182.

[13] A. Gorbenko and V. Popov, The Far From Most String Problem, Applied Mathematical Sciences, 6 (2012), 6719-6724.

[14] A. Gorbenko and V. Popov, Multi-agent Path Planning, Applied Mathematical Sciences, 6 (2012), 6733-6737.

[15] A. Gorbenko and V. Popov, On the Problem of Placement of Visual Landmarks, Applied Mathematical Sciences, 6 (2012), 689-696.

[16] A. Gorbenko and V. Popov, The c-Fragment Longest Arc-Preserving Common Subsequence Problem, IAENG International Journal of Computer Science, 39 (2012), 231-238.

[17] A. Gorbenko and V. Popov, Task-resource Scheduling Problem, International Journal of Automation and Computing, 9 (2012), 429-441.

[18] A. Gorbenko and V. Popov, The Hamiltonian Alternating Path Problem, IAENG International Journal of Applied Mathematics, 42 (2012) 204-213.

[19] K. Iwama and S. Miyazaki, SAR-variable complexity of hard combinatorial problems, IFIP Transactions A, Computer Science and Technology, 1 (1994) 253-258.

\section{Received: December 3, 2012}

